\title{
SAR Imagery Segmentation by Statistical Region Growing and Hierarchical Merging
}

\author{
Eduardo A. Carvalho ${ }^{1} \quad$ Daniela M. Ushizima ${ }^{2, *} \quad$ Fátima N. S.Medeiros ${ }^{1}$ \\ Charles I. O. Martins ${ }^{1}$ Regis C. P. Marques ${ }^{3}$ \\ ${ }^{1}$ Department of Teleinformatics Engineering, Federal University of Ceará, Fortaleza-CE, Brazil \\ ${ }^{2}$ Computational Research Division, Lawrence Berkeley National Laboratory, Berkeley-CA, USA \\ ${ }^{3}$ Federal Institute of Education, Science and Technology, Ceará, Fortaleza-CE, Brazil \\ eduardo@deti.ufc.br, dushizima@lbl.gov, fsombra@ufc.br, charlesiury@gmail.com, regismarques@cefet-ce.br,
}

\begin{abstract}
This paper presents an approach to accomplish synthetic aperture radar (SAR) image segmentation, which are corrupted by speckle noise. Some ordinary segmentation techniques may require speckle filtering previously. Our approach performs radar image segmentation using the original noisy pixels as input data, eliminating preprocessing steps, an advantage over most of the current methods. The algorithm comprises a statistical region growing procedure combined with hierarchical region merging to extract regions of interest from SAR images. The region growing step oversegments the input image to enable region aggregation by employing a combination of the KolmogorovSmirnov (KS) test with a hierarchical stepwise optimization (HSWO) algorithm for the process coordination. We have tested and assessed the proposed technique on artificially speckled image and real SAR data containing different types of targets ${ }^{1}$.
\end{abstract}

\section{Introduction}

The continuous demand for SAR images interpretation by automatic procedures is a key issue in the remote sensing field due to the increasing amount of data generated by SAR systems recently. The task of interpreting SAR images involves identification of the structures found in the scene using image segmentation algorithms, usually based on image domain features compared to known attributes or patterns (ground truth).

SAR imagery segmentation is severely penalized by

\footnotetext{
${ }^{1}$ This version contains errors. The correct version of this paper can be retrieved from Digital Signal Processing, Elsevier 2010
}

the presence of coherent noise, known as speckle, that degrades fine details and edges of the objects present in the scene. Speckle appears due to interference of coherent waves, which bounce off the targets in the radar illuminated area, consequently generating a grainy image, containing pixels with high gray level fluctuations.

The image understanding automation of speckled images as SAR and ultrasound requires some segmentation step such as edge detectors, region growing, thresholding and other algorithm, but they can fail to segment such images due to the influence of the speckle noise, usually ignored by such algorithms and its statistics. These methods often require filtered images to provide acceptable image segmentation results as in Kuan $[1 ; 2 ; 3$ ? ]. Also, combinations of filters can provide speckle smoothing with edge preservation as in [4]. Nonetheless, speckle filtering is extremely sensitive in real applications, subjected to data modifications, resulting in edge smoothing, elimination of small structures and artifacts [5].

Edge-based methods as Sobel, Canny detectors, Roberts, Prewitt, LoG are usually not effective for SAR images since they use gradient detectors based on the difference between neighboring pixel values, which fails in the presence of speckle noise. Improvements to edgebased methods in Moigne and Tilton [6] integrated edges produced by a Canny detector with region growing aiming to minimize the Hausdorff distance between edge maps and region boundaries. This algorithm performed well in Landsat Thematic Mapper (TM) images, but requires a more suitable edge detector to be useful in SAR images. Also, edge strength estimation in speckled images SAR can be accomplished by using ratio-based edge detectors which evaluate transition between uniform areas. 
Improved edge detectors customized for SAR image segmentation utilizes a sliding window over the image to calculate the ratio of means given a neighborhood definition. These detectors compare the obtained ratio with a threshold that is calculated based on a probability of false alarm $(p f a)$ to determine whether an edge is present or not $[7 ; 8 ; 9 ; 10]$, an useful criterium considered in the current paper.

Yu and Clausi [11] combined edge information and region growing to design an improved segmentation algorithm for ice floes segmentation and classification, regarding a Markov random field context model to map edge strength. Their cost function suppresses the merging between parts of different objects that have weak boundaries, i.e. the method penalizes more the weak edges than the strong ones. The number of iterations to merge adjacent regions depend on the oversegmentation, which is calculated using watershed transformation [11]. Our concern about this process is the gradient operation before the watershed calculation, which is mostly unstable for noisy image segmentation.

Alternatively, we propose an oversegmentation moderated by a coefficient of variation, aiming at reducing the computing time. Our initialization calculates a smaller number of segments for a SAR image given the statistics following the homogeneity criteria, as discussed in 2 .

Recently, Bernad et al. [12] designed an interactive tool to simplify SAR image analysis. First, it segments the image into homogeneous regions based on speckle statistics, followed by region labeling into classes of significance to a given application, a semi-automatic process. The segmentation scheme was accomplished by following the algorithm proposed in Galland et al.[13] which consists in a new minimum description length (MDL) approach based on a polygonal grid partition of the image for segmentation of a speckled image. From the homogeneous regions of the image, they derive a set of measures such as the coefficient of variation defined by the ratio of mean to standard deviation to be computed over all the pixels of each region and between regions. Their contributions are relevant and worthed as a comparison method when the systems presents both a unique available channel SAR image and relies on user-interaction.

Circumventing the restriction of semi-automatic systems, we propose a statistical region growing algorithm that takes into account an hierarchical decomposition of the image. Different from threshold and edge-based methods, region growing methods can incorporate regional features but it still remains the problem of establishing the initial regions, finding reasonable region descriptors or features to enable proper merging of sim- ilar regions. The strategy used in this paper is to aggregate statistically homogeneous regions to create new segments. We use an initial set of regions to carry out automatic segmentation by merging using moments (MUM)[14].

Several papers address SAR image segmentation based on region growing and hierarchical model $[15 ; 11]$ and previous work on Hierarchical Stepwise Optimization by Beaulieu and Goldberg [16], known as HSWO, remains an effective approach to SAR image segmentation $[17 ; 15]$. HSWO exploits the idea of hierarchy in segmentation to provide a step-wise merging of the segments of the previous image partition. Yang et al. proposed improvements to HSWO, introducing supervised SAR image segmentation based on a region-based half-tree hierarchical model by applying multiresolution analysis and supervised MPM (maximization of posterior marginal estimation) algorithm [15]. Similarly, we consider hierarchical analysis to merge oversegmented regions in the image. The proposed method presents an original algorithm to perform segmentation of SAR images without considering a priori information regarding the structures to be found in the image.

This paper is organized as follows. Section 2 presents the theoretical background to support the methodology development and understanding. Section 3 introduces the proposed method for speckled image segmentation starting with the SAR image decomposed into a great amount of adjacent labeled regions by using a modified region growing algorithm. Section 4 presents the results of the proposed method applied to synthetic and real SAR including performance assessment. Finally, in Section 5, we conclude the present paper and consider further works that can be developed.

\section{Theoretical Background}

\subsection{Speckle Statistics and Image Model}

The influence of speckle noise compromises valuable information on SAR images. This noise follows the multiplicative model, which states that the corrupted backscattered signal, $z$, can be expressed as a product of the speckle noise $n$ and the terrain backscatter $x$.

Several distributions could describe the backscatter, aiming at modeling different types of terrain and their characteristic degrees of homogeneity. Table 5 displays the distributions concerning SAR data returns, combining speckle and different backscatter models when adopting that speckle noise follows a Square Root of Gamma distribution. Regarding sensor parameters as wavelength, angle of incidence, polarization, among others, pasture is more homogeneous than forest, which, in turn, is more homogeneous than urban 
areas $[18]$

Table 1. Return distributions for different radar surfaces under the multiplicative model [18].

\begin{tabular}{lll}
\hline Region Type & Noiseless Scene $(x)$ & Return $(z)$ \\
\hline Homogeneous & $\mathcal{C}(\sqrt{\beta})$ & $\Gamma^{1 / 2}(L, L / \beta)$ \\
Heterogeneous & $\Gamma^{1 / 2}(\alpha, \lambda)$ & $\mathcal{K}_{\mathcal{A}}(\alpha, \lambda, L)$ \\
+Heterogeneous & $\Gamma^{-1 / 2}(\alpha, \gamma)$ & $\mathcal{G}_{A}^{0}(\alpha, \gamma, L)$ \\
General case & $\mathcal{N}^{-1 / 2}(\alpha, \gamma, \lambda)$ & $\mathcal{G}_{A}^{0}(\alpha, \gamma, \lambda, L)$ \\
\hline
\end{tabular}

The reader may refer to [18] for more details about the these distributions to model radar data. The parameters $\alpha, \beta, \lambda$ and $\gamma$ are calculated using the mean gray level $(\bar{z})$ of the synthetic image segments and $L$ (number of looks) [19]. The parameter $\alpha$ and $\lambda$ indicate the degree of roughness and scale, respectively.

In this paper we adopted only two degrees of homogeneity for the terrain backscatter in radar images, homogeneous and heterogeneous areas, regarding the coefficient of variation as in $[20 ; 21 ; 22]$.

Assuming that SAR image has been conveniently processed to eliminate interpixel correlation, the observed coefficient of variation $(C V)$ defines the standard deviation to the mean ratio of a set of pixels in a homogeneous area, namely:

$$
C V=\frac{\sigma_{z}}{\bar{z}} .
$$

The coefficient of variation can be used as an homogeneous test, so if the terrain backscatter has constant average intensity, then $C V \simeq \sigma_{n}$ but if it varies, $C V \geq \sigma_{n}$, for the speckle standard deviation $\sigma_{n}[22]$. For amplitude SAR images, an area is considered homogeneous if $\sigma_{n}$ equals $0.5227 / \sqrt{L}$, where $L$ is the number of looks [23]. The unilateral limit used by $[20 ; 21 ; 22]$ admits a region as statistically homogeneous if

$$
C V \leq \sigma_{n}
$$

We adopt an automatic procedure to estimate speckle standard deviation from SAR images as in Frulla et al [24], which observed that the $C V$ should be equal to the theoretical speckle standard deviation according to the number of looks of the SAR image for fully developed speckled images. However, the coefficient of variation measured over homogeneous regions on the images may differ from theoretical values due to several reasons such as the inherent image variations and the size of the selected sample.

We circumvent this problem by combining the coefficient of variation to the proposed region growing method, which restricts the calculation of $C V$ to homogeneous zones. The statistical analysis of the speckle noise in homogeneous regions by using $C V$ produces adaptive segment formation in the initial partition of the image. Henceforth, a similarity criterion for pixel clustering moderate the merging of the small disjoint segments, using information collected from the original data.

\subsection{Region Growing and merging}

After defining an initial set of small areas, region growing algorithms iteratively merge pixels into sets, according to a similarity criteria as a given range of gray scale levels. It starts by choosing an arbitrary seed pixel to be compared with a neighborhood, followed by the addition of similar neighboring pixels, increasing the size of this set, which defines a segment. When a segment stops growing, we have a region and another seed pixel which does not yet belong to any region is chosen to start the process again. Region growing algorithms are bottom-up methods, addressing a similarity rule to join pixels to regions. A drawback of this method relies on the definition of a similarity rule to iteratively segment regions according to a domain, as well as the number of seeds to use, specially when applied to raw SAR data.

An approach to refine the region growing segmentation results is to use a hierarchical region merging algorithm as the hierarchical stepwise optimization algorithm (HSWO) [25; 26], which compares spatially adjacent regions to merge them in an iterative process. The inputs are an image and its preliminary segmentation into $\mathrm{N}$ labeled regions, given that all the pixels have some label and each region is connected. Spatially adjacent regions are compared and merged with most similar regions until the algorithm either reaches a specified number of regions or a dissimilarity between the most similar pair of spatially adjacent regions reaches a specified threshold. Although the algorithm assumes that each iteration is statistically, the HSWO method [16] still produces excellent results [27].

We summarize the algorithm in [25] bellow:

\section{The HSWO Algorithm [25].}

I. Begin
i. $P^{0}=\left\{S_{1}, S_{2}, \ldots, S_{N}\right\}$ (initial partition);
ii. $k=0$ e $m=N$;
iii. Calculate $D_{i}$ and $B_{i}$ for $S_{i} \in P^{0}$;
iv. Calculate $C S=\left\{C_{i, j} \mid S_{j} \in B_{i}\right.$ e $\left.i \neq j\right\}$.

II. Merge the two similar segments 


$$
\begin{aligned}
& \text { i. } k=k+1 \text { and } m=m+1 ; \\
& \text { ii. Find } C_{u, v}=\min \left\{C_{i, j}\right\} ; C_{i, j} \in C S ; \\
& \text { iii. } P^{k}=\left(P^{k-1} \cup\left\{S_{m}\right\}\right) \cap \overline{\left\{S_{u}, S_{v}\right\}} \\
& \text { iv. Calculate } D_{m} \text { from } D_{u} \text { and } D_{v} ; \\
& \text { v. } B_{m}=\left(B_{u} \cup B_{v}\right) \cap \overline{\left\{S_{u}, S_{v}\right\}} ; \\
& \text { vi. } \forall S_{j} \in B_{m}, B_{j}=\left(B_{j} \cup\left\{S_{m}\right\}\right) \cap \overline{\left\{S_{u}, S_{v}\right\}} ; \\
& \text { vii. } C S=\left(C S \cup\left\{C_{m, j} \mid S_{j} \in B_{m}\right\}\right) \cap \\
&\left\{C_{i, j} \mid i, j=u \text { or } v\right\}
\end{aligned}
$$

III. Stopping condition

i. Stop if no more mergers are required.;

ii. Otherwise, go to step II.

A shortcoming of the HSWO algorithm is its dependency on a predefined number of segments or iterations that must be specified by the user. To circumvent the lack of a proper stop criterion for the HSWO algorithm, our approach consists in using the HWSO to coordinate the election of the two most similar regions in the RAG, whilst effective merging is done via a hypothesis test. It is desirable that the election of the two most similar regions in the RAG is performed with proper descriptors to formulate a convenient cost function. Once elected the two regions in the RAG according to the minimum cost, we apply the two-samples Kolmogorov-Smirnov hypothesis test to effective provide region fusion.

\section{Proposed Method}

We propose a framework to segment SAR images using a statistical region growing algorithm, which can be summarized in 3 main steps: (1) choice of the seed pixels (or regions), then (2) neighborhood analysis according to a similarity rule, in our case, the coefficient of variation. Finally, the step (3) guarantees a forward inclusion of adjacent pixels to grow the seed regions for those pixels that satisfy the similarity rule. The steps (1) and (2) are repeated until there are no more adjacent pixels to be included in a seed region. The process restarts with another seed region and iterates until there are no more regions to merge, as illustrate in the flowchart of Fig.??

Before starting the statistical region growing process, the image pixels are initially labeled, defining a label matrix. A $3 \times 3$ window centered at a random position $(i, j)$ defines a seed homogeneous segment if this window lies over a statistically homogeneous zone of the radar image, according to Equation (1). If the homogeneity criterion is satisfied, all the 8-connected pixels to $(i, j)$ delimited by the window have their labels updated to the same as the central pixel.

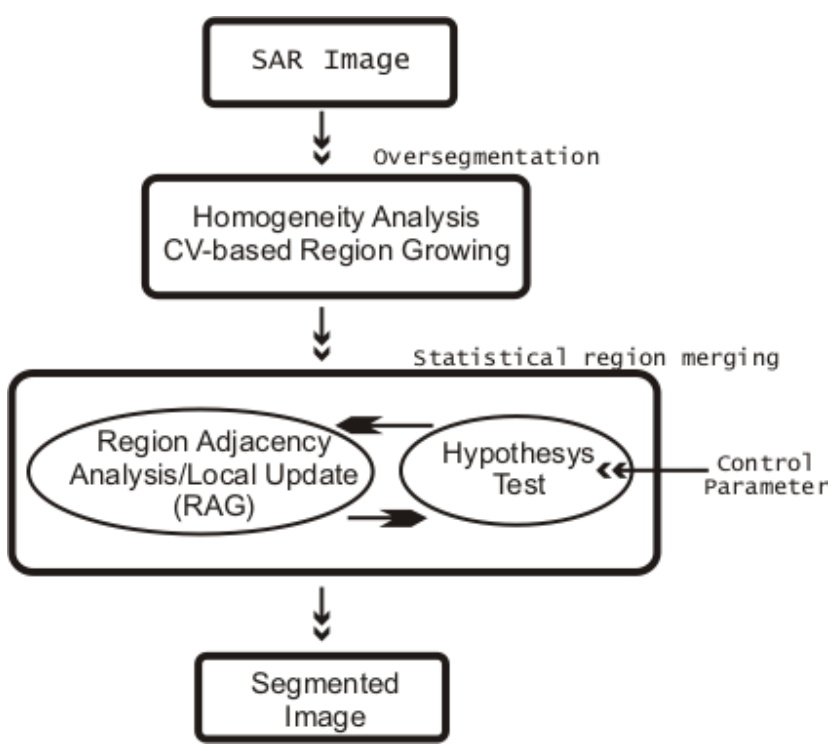

Figure 1. The proposed block diagram of the segmentation method.

Next step is to determine the pixels adjacent to this initial region, which are randomly selected to be inserted into the region temporarily. Each time a candidate pixel is included in this region, a new coefficient is calculated using all the pixels of the region and only after a comparison with $C V \leq \sigma_{n}+\delta$, the algorithm decides whether a pixel is accepted or not. If the candidate pixel satisfies the $C V$ condition, its label assumes the region label.

The compensating factor $\delta$ is taken into account for small deviations of the ratio $C V=\sigma_{z} / \bar{z}$ from the speckle standard deviation $\sigma_{n} . \delta$ is derived from [22] and it is established as:

$$
\delta=\sigma_{n} \sqrt{\frac{1+2 \sigma_{n}^{2}}{2 N}},
$$

where $N$ is the size of the region.

In our approach we use $\delta$ multiplied by a small constant $\eta$ that penalizes the non-linear component of the $C V$ to create a general threshold $T$, to be compared with $C V$ and finally decide whether a candidate pixel still keeps the segment statistically homogeneous. This threshold is given by:

$$
T=\sigma_{n}\left(1+\eta \sqrt{\frac{1+2 \sigma_{n}^{2}}{2 N}}\right),
$$

with $\eta$ experimentally set in this work to 0.075 .

If there are no more adjacent pixels to test the similarity condition $\sigma_{z} / \bar{z} \leq T$, then a new position $(i, j)$ is randomly selected in the image to be the central pixel of the window and the inclusion of candidate pixels follows the scheme as described earlier. 
Regions aggregation is obtained by applying a nonparametric hypothesis test using pixel information of two similar regions elected in a hierarchical tree. The test considers a cost function based on the ratio of averages of a pixel cluster along the common frontier of two segments. The merging of the two most statistically similar regions are based on a region adjacency graph (RAG)[16; 26; 28], previously generated from the initial segmentation.

This graph or hierarchical tree contains the neighboring relations among the segments occurring in the initial partition, whose structure is locally updated each time merging is accomplished. The fusion degree for the segments during the merging stage is ranked by the significance level $\left(p_{0}\right)$ of the Kolmogorov-Smirnov hypothesis test. Whenever two minimum cost segments merge, the RAG topology is locally updated. Costs are calculated according to those new adjacency relations. The merging process is iterative and stops when there are no more regions to merge.

\subsection{Constraints on Statistical Region Growing}

The coefficient of variation $(C V)$ is an efficient criteria to test homogeneity, so we use it to guide the region growing, but it requires constraints to be useful in our framework as (i) minimum and maximum size of regions, (ii) label attribution control for the segments, (iii) control of visited pixels and (iv) identification of grown regions.

We establish the minimum size of a segment to be the minimum window size, i.e. nine pixels, which can grow to the maximum size depending on the $C V$ value for the neighboring pixels. If $(C V)$ value is low, the region surrounding the central pixel must be homogeneous. Otherwise, it is likely there exists an edge in the segment.

The proposed algorithm starts with an oversegmentation by establishing the maximum size to be maxpixels $=15$ pixels for the initial region growing process. The maxpixels is an empirical parameter of the region growing algorithm to preserve as much structural features (e.g. edges or lines) or strong scatterers as possible. In homogeneous areas (e.g. crops) where only texture speckle exists, the coefficient of variation $(C V)$ has a precise value used as an indicator of region homogeneity. Moreover, it is sensitive to the presence of a contour independently of its direction. In our experiments this empirical number favored the extraction of the most likely real boundaries in the image separating textured fields.

Larger windows cause loss resolution for segment borders. Limiting the maximum size of regions during pixel insertion aims at minimizing the inclusion of pixels that belong to areas with different textures (e.g.edge)

For instance, SAR images comprising large crop fields may have the initial segments formation with a greater number of pixels $(20,25, \ldots)$. From this scheme, we aim at minimizing the chance of including pixels belonging to other adjacent regions. On the other hand, the segments of single pixel must be tested in order to include new pixels. These segments may increase until they reach the maximum size maxpixels if their adjacent pixels satisfy the similarity rule.

If the limit is not reached during the region growing process, it means that the surrounding candidate pixels have not satisfied the condition $C V \leq T$. To illustrate this idea, we show on the region labeled 42 on the middle-left of Fig. 2.

Fig. 2 depicts situations where segments and their adjacency present (a) predefined regions, (b) non visited pixels and predefined segments and (c) some unvisited pixels in the interior or located in the borders of an image under analysis.

\begin{tabular}{|l|l|l|l|l|l|l|l|l|l|l|l|}
\hline 01 & 02 & 14 & 14 & 14 & 14 & 07 & 08 & 09 & 10 \\
\hline 14 & 14 & 14 & 14 & 14 & 14 & 17 & 18 & 19 & 20 \\
\hline 42 & 14 & 14 & 14 & 14 & 14 & 48 & 48 & 29 & 30 \\
\hline 42 & 42 & 42 & 45 & 45 & 45 & 48 & 48 & 48 & 40 \\
\hline 42 & 42 & 42 & 45 & 45 & 45 & 48 & 48 & 48 & 48 \\
\hline 42 & 42 & 42 & 45 & 45 & 45 & 48 & 48 & 48 & 48 \\
\hline 42 & 42 & 75 & 75 & 75 & 75 & 48 & 68 & 69 & 48 \\
\hline 71 & 72 & 75 & 75 & 75 & 75 & 75 & 75 & 79 & 80 \\
\hline 81 & 82 & 83 & 75 & 75 & 75 & 75 & 99 & 99 & 99 \\
\hline 113 & 92 & 93 & 94 & 95 & 96 & 97 & 99 & 99 & 99 \\
\hline 113 & 113 & 113 & 113 & 113 & 106 & 107 & 99 & 99 & 99 \\
\hline 113 & 113 & 113 & 113 & 113 & 113 & 117 & 118 & 119 & 120 \\
\hline 121 & 113 & 113 & 113 & 125 & 126 & 127 & 128 & 129 & 130 \\
\hline
\end{tabular}

Figure 2. Label matrix with predefined segments (14, $42, \ldots, 99$ and 113) and unvisited pixels (white squares).

The region labeled 45 is an example of predefined pixels because its neighboring regions are already defined as 14, 42, 48 and 75, therefore region 45 can not grow anymore and it will not reach the maximum value (maxpixels) in this iteration. In this stage, it is allowed the inclusion of single pixels at once. Region 42 and 45 are cases of grown regions which did not reach the maximum number of pixels allowed maxpixels $=15$.

The situation (b) arises due to the size of the window to start at $3 \times 3$, well exemplified in region 99 , on 
the bottom-right of Fig.[? ]. This small region must include the pixels labeled as 79, 80, 97, 107,.., 120 (surrounding pixels), as long as they satisfy the homogeneity criterion $\sigma_{z} / \bar{z} \leq T$.

Situation (c) occurs for all unvisited pixels in the image that present other pixels or predefined regions as neighbors, when there are no more positions for the window to be installed. These pixels create holes among small regions and they must be included into the existing regions, since they satisfy other requisites.

The final step of the proposed region growing algorithm consists in eliminating single pixel regions. The strategy to allocate the remainder unvisited (isolated) pixels is accomplished by the flowchart displayed in Fig. 3.

Besides the label matrix, represented in Fig.[? ], there is a 'mask' (or control matrix) that keeps track of pixel positions as they are visited, preventing the inclusion of regions formed previously. The mask and label matrix analysis enables the algorithm to track isolated and unvisited pixels, aiming at inserting them into the existing regions. Pixel moving is achieved by verifying the changes on the coefficient of variation after a temporary insertion of a candidate pixel into each of this neighboring regions. At this stage, the amount of pixels per segment may extrapolate the value maxpixels.

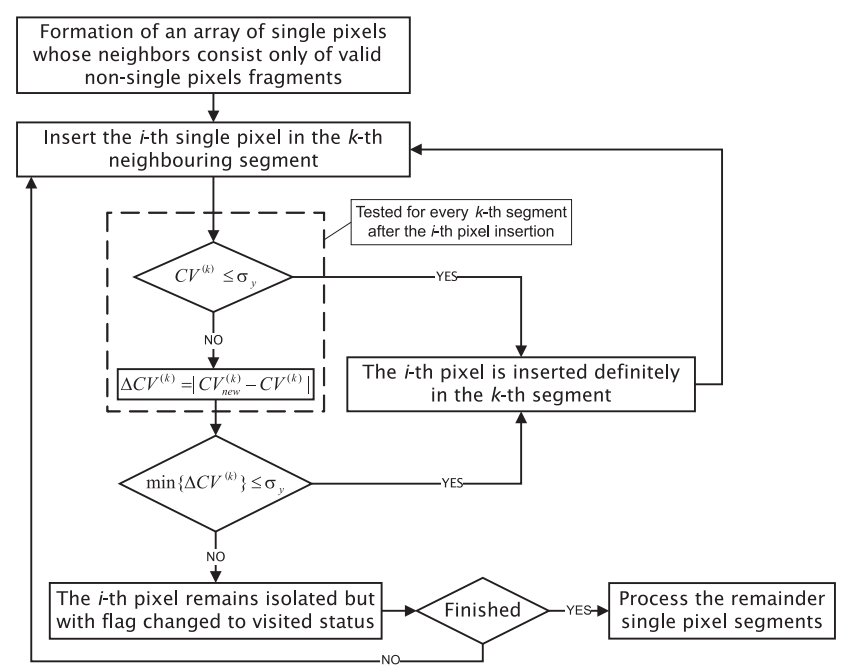

Figure 3. The proposed approach to allocate unvisited pixels during the oversegmentation step.

Speckle statistics analysis of homogeneous regions discards regions comprising only one pixel. If the procedure fails to allocate the isolated pixels of the builtup array (or list to process), the remainder one pixel regions (now with visited status in the mask) are forcefully inserted into the existing regions, according to the following constraints: a. All of the unaccepted pixels labels are stored in an array. For each entry of this array, only those neighboring segment labels that do not exist in this array are retained. This guarantees that an unaccepted pixel is not merged with another single pixel, but inserted in an already existing region;

b. An unaccepted pixel is chosen arbitrarily and it is inserted in each adjacent region, whose coefficients are updated;

c. The unaccepted pixel is definitely sent to the region whose the calculated coefficient of variation is less than $\sigma_{n}$. On the other hand, it is accepted into that regions, whose pixel insertion produced the smallest coefficient alteration.

The initial regions formed by applying the similarity measure based on the $C V$ are now used to receive the remainder single pixels, which were not included during the homogeneity step (unvisited pixels). This is performed by a new $C V$-based testing, where a single pixel (or hole) is put into a neighboring region only if it does not affect its homogeneity.

These verifications are executed until there are no more single pixels, where the described constraints are based on the coefficient of variation analysis and on the mask (label controlling matrix) verification. Fig. 4 exhibits a detail of an oversegmented SAR image produced by the region growing algorithm based on the coefficient of variation analysis.

It is worth noting that if we perform a regular tessellation of the image depicted in Fig. 4(a) by applying a $2 \times 2$ grid, there would exist 10,000 blocks, what is a considerable amount of four pixel regions. Although the grid division process (image division by windowing, $2 \times 2,3 \times 3, \ldots)$ is quite simple and direct, region merging is accomplished at a great time expense. Besides that, descriptors like the gray mean value and variance of the pixels that compose the window of analysis may be biased due to the noise influence, since the radiometric values vary strongly from one pixel to another in certain image areas.

\subsection{The Proposed Cost Function}

This section addresses the problem of finding out suitable descriptors to establish a similarity metric between segments and proposes a cost function based on the border detection theory. To segment SAR images under this approach, the mean gray level of the segments may not satisfactorily be applied on the region merging, due to the speckle influence mainly in small samples. In edge detection applications, it is commonly used the ratio of averages to infer about the presence 


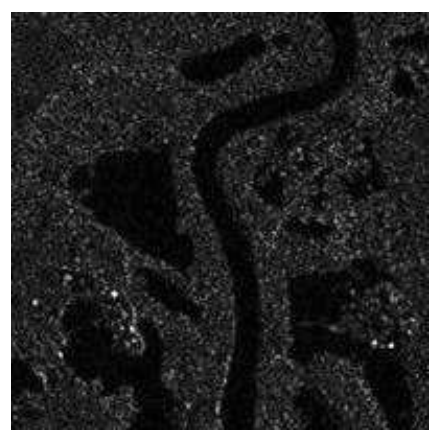

(a)

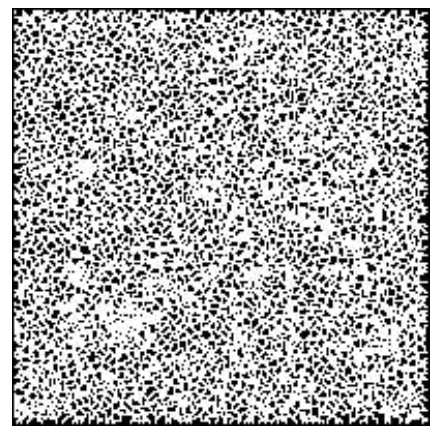

(b)

Figure 4. (a) A $200 \times 200$ detail of a SAR image and (b) edge map comprising 3982 regions created by the proposed statistical region growing.

of edges, instead of the difference of means, to provide a constant false alarm ( cfar) detector [29].

Our RAG scheme was inspired by [14] that considered the size and the length of the common frontier along the regions created by the region growing process. Differently from the aforementioned work we use the ratio of the averages of border pixels combined with size and length features.

Fig. 5 depicts two regions interface, where pixels labelled as ' $O$ ' and ' $x$ ' represent the border pixels, whereas ' + ' and ' $\oslash$ ' are their respective neighborhoods.

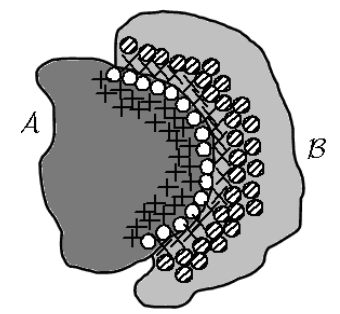

Figure 5. Local pixels along the frontier of two regions.

The proposed cost criterion to generate the region adjacency graph is defined as:

$$
C_{A, B}=\frac{\min \left(N_{A^{\prime}}, N_{B^{\prime}}\right) \cdot r}{Q_{A, B}^{2}},
$$

where $N_{A^{\prime}}, N_{B^{\prime}}$ stand for the population (number) of pixels along or in the surroundings of each border. $Q_{A, B}$ is the common border length, in pixels, and $r$ is given by:

$$
r=1-\min \left\{\frac{\bar{z}_{A^{\prime}}}{\bar{z}_{B^{\prime}}}, \frac{\bar{z}_{B^{\prime}}}{\bar{z}_{A^{\prime}}}\right\},
$$

that uses the noisy border pixels ' $x$ ' and ' $O$ ' to calculate the mean values $\bar{z}_{A^{\prime}}$ and $\bar{z}_{B^{\prime}}$.

Equation (5) points that the retention of the smallest region size implies the inclusion of small segments to minimize their existence at the end of the fusion process. The local ratio of averages considers the influence of border pixels, instead of those located far away from the common border of two segments.

The influence of the factor $Q_{A, B}$ considers the length of the common border. The greater the border, the greater the chance two regions $A$ and $B$ to be elected in the RAG, when there is statistical similarity between them. For SAR images, the mean gray level considering all the pixels of a segment is not suited as a descriptor for region similarity, a problem known as the mean gray value degradation [30].

\subsection{The Region Merging via Statistical Hypothesis Testing}

The hierarchical stepwise optimization (HSWO) algorithm achieves a substantial reduction in the number of regions. When the merging of two regions occurs, no recalculations for the cost function of all the nodes in the hierarchical tree are needed. This event is locally performed, based on the new partition methodology which is used here. Moreover, different cost functions can be adopted in order to elect the most similar regions of a region adjacency graph (RAG), depending on the features of the image under analysis.

To effective accomplish region fusion of a pair of similar regions elected by the HSWO algorithm, we have applied the two-samples Kolmogorov-Smirnov (KS) [31; 32] hypothesis test. The proposed algorithm combines the KS test and the HSWO algorithm in the region merging step to overcome the shortcomings of the hierarchical stepwise approach. User interaction is done by a parameter called significance level of the test, $p_{0}$. This variable is a small probability $(0.1,0.001$, $0.0001, \ldots$ ) threshold to be compared with the hypothesis test probability to infer whether two regions can be merged or not. By means of the parameter $p_{0}$, different number of segments are obtained after concluding region merging. 


\section{Experimental Results for Synthetic and Real SAR Images}

In this section, the performance assessment of the proposed segmentation technique is accomplished, according to qualitative and quantitative aspects. The tests were carried out with artificially and real SAR images. Fig. 6 shows artificially contaminated versions of the $k k$ and imgcartoon images for different number of looks $(L=1,3$ and 5$)$. Fig. 7 and Fig. 8 display the edge maps for the segmented speckled versions of $k k$ and imgcartoon images, respectively.

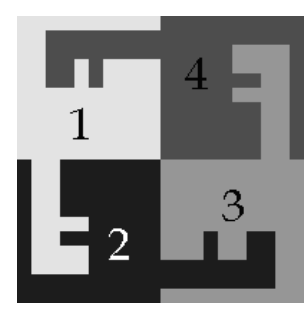

(a)

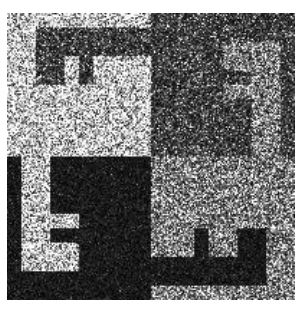

(b)

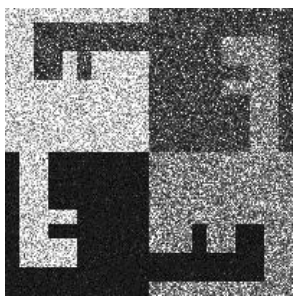

(c)

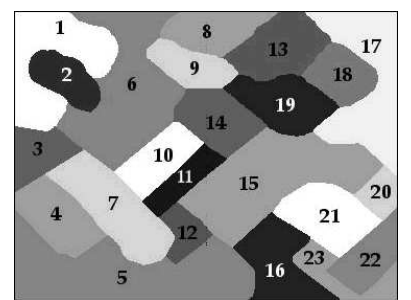

(d)

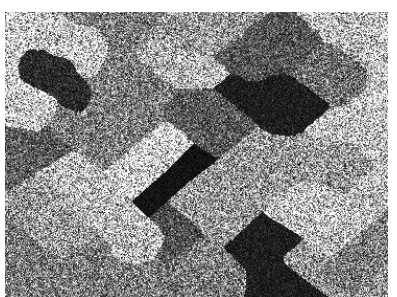

(e)

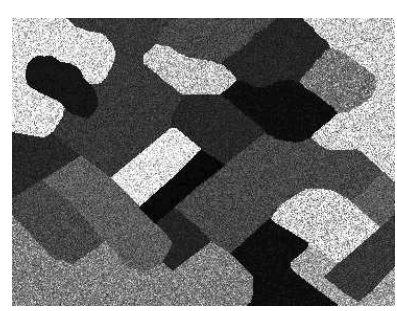

(f)

Figure 6. Synthetic speckled versions of (a) kkimage for (b) 1 look, (c) 3 looks and (d) imgcartoon image for (e) 3 looks and (f) 5 looks.

The segmentation results were compared with the ground truth or the reference segmentation of $k k$ and imgcartoon, according to the measures Totgof and Ajbrd $[19 ; 33 ; 34]$. These measures are in the interval $[0,1]$, being equal to unity whenever two regions (the reference and the segmented counterpart) fit perfectly. Totgof permits to infer the global performance of a segmentation algorithm, whereas Ajbrd measures the fitness of the ideal limits of the objects of the segmented ground truth image compared to the real borders produced by the noisy image segmentation.

In addition, to achieve a comparative analysis, it was still used the region growing implementation provide by software ENVI 3.4. The light gray contours refer to the ideal edges and the black ones correspond to the effective edges provided by our method.

One can observe that the algorithm failed in detecting correctly the transition areas for some regions, e.g. the first quadrant in $k k$ image as shows the Fig. 7(a) and the regions labelled 4, 5 and 7 of imgcartoon shown in $8(\mathrm{a})$. However, in many points the contours are well overlaid, what indicates that the algorithm succeeded in preserving edges.

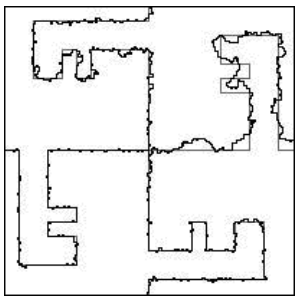

(a)

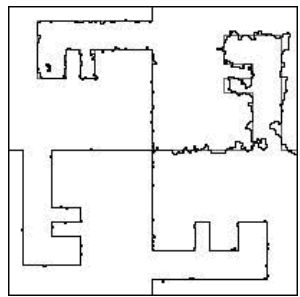

(b)

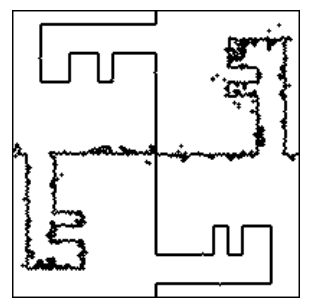

(c)

Figure 7. Edge maps for the segmented $k k$ image (a) with 1 look and $p_{0}=10^{-6}$ and (b-c) with 3 look, for $p_{0}=10^{-5}$ and by using region growing implementation at ENVI software, respectively. Gray lines: ideal edges; black lines: calculated edges.

The segmentation performance evaluation is carried out for $L$ looks and $p_{0}$ equal to $10^{-5}$ and $10^{-6}$, respectively. We also investigate the influence of the $p_{0}$ parameter (significance level) of the KS test, in the final number of regions.

For each value of this parameter, the simulated images ( $L=1,3$ and 5 looks) were segmented. By means of 


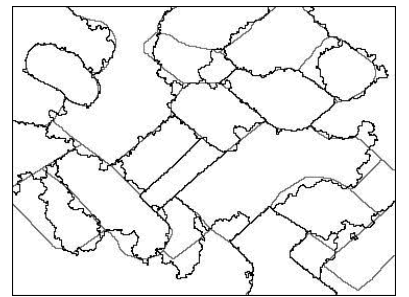

(a)

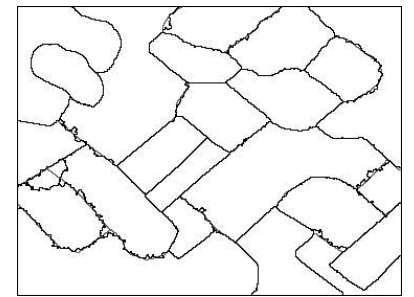

(b)

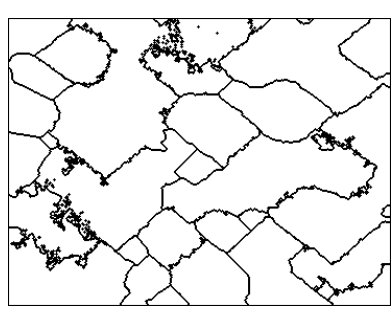

(c)
Figure 8. Edge maps for the segmented imgcartoon image (a) with 1 look and $p_{0}=10^{-6}$ and (b-c) with 5 look, for $p_{0}=10^{-6}$ and by using region growing implementation at ENVI software, respectively. Gray lines: ideal edges; black lines: calculated edges.

discrepancy measures one can infer which results better approximate the ideal segmentation for the test images. We also investigate whether the method performs well for non homogeneous radar images or scenes that present a mixture of different reflectivity surfaces.

\subsection{Segmentation Results Assessment}

The measures Totgof for $k k$ and imgcartoon segmentation results, with $p_{0}=10^{-5}$ and $10^{-6}$, respectively, were calculated for different number of looks. Both images presented values that indicate good edge preservation for most part of the segments for both artificially contaminated images. The speckle noise is more intense in 1-look synthetic and real SAR images. For that reason the $k k$ and imgcartoon 1-look simulated images presented the lowest values for the Totgof measures when compared with the multiple looks simulated versions. Thus, the algorithm achieved the best segmentation results for the 3 -looks $k k($ Totgof $=0.9814)$ and 5-looks imgcartoon (Totgof $=0.9841)$ images. It is worth noting that the nearer the value of Totgof to unity, the better the overall reconstruction of the segments related to the ground truth image.

The results of edge fitness Ajbrd per segment are indicated in the graphs illustrated in Fig. 9, where numbers $1,2, \ldots, 23$ indicate the region labels of the ground truth image, whereas the vertical axis stands for the measure values.

For the speckled 3-looks $k k$ image, the algorithm

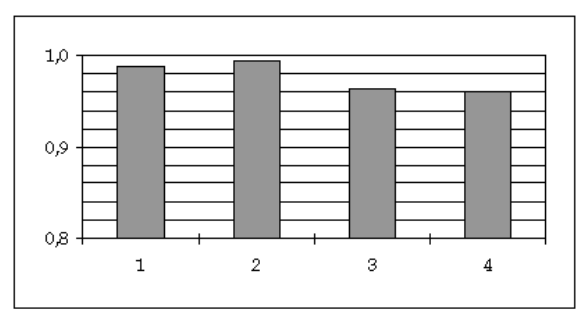

(a)

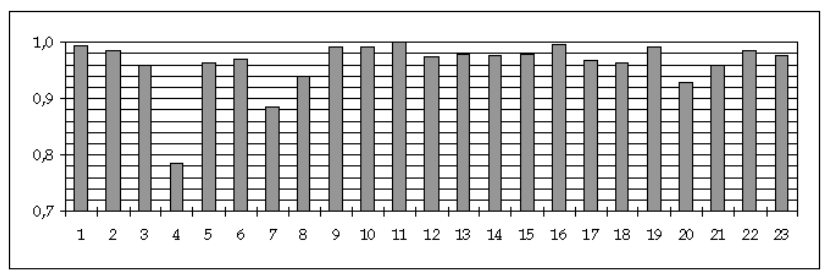

(b)

Figure 9. Measures per segment of Ajbrd for (a) $k k$ (3 looks, $p_{0}=10^{-5}$ ) and (b) imgcartoon (5 looks, $p_{0}=10^{-6}$ ).

failed in detecting the true edges of the ' $\mathrm{F}$ ' pattern in the first quadrant of Fig. 7(b), resulting in low values for the measure Ajbrd in regions 3 and 4 (Fig. $9(\mathrm{a}))$. Nevertheless, edges in this segmented $k k$ image are closer to the true one (ground truth) than the results presented in Fig. 7(a). The segmented 5-looks imgcartoon image with $p_{0}=10^{-6}$ has created a region surrounded by regions labelled 3, 4 and 7, which diminished the values of Ajbrd for regions 4 and 7 . One can perceive it in Fig. 9(b), related to the edge map of Fig. 8(b).

\subsection{Real SAR Images}

The proposed segmentation algorithm was applied to the real SAR images shown in Figs. 10(a), 11(a) and $12(\mathrm{a})$. The subimage comprising $512 \times 512$ pixels shown in Fig. 10 was taken from the image Caçapava, acquired by Radarsat-1 from the region of the Paraíba Valley, state of São Paulo, Brazil. In this image, the Paraíba river stands out with lakes along both margins and the diagonal large strip in the middle left is a rice culture. Fig. 10(b) illustrates the segmented version of this image with $p_{0}=10^{-6}$.

Fig. 11 presents the segmentation result for the SAR imagePedazo. It is an amplitude real image with measured 2,21 looks. It was taken from a region of Munich, in Germany. The bright structures in the left superior part of this image were preserved and it is possible to observe edge delineation in the dark regions. These regions are homogeneous zones surrounded by wood areas and urban spots. Other remaining fragments consist of detected small regions that present considerable 


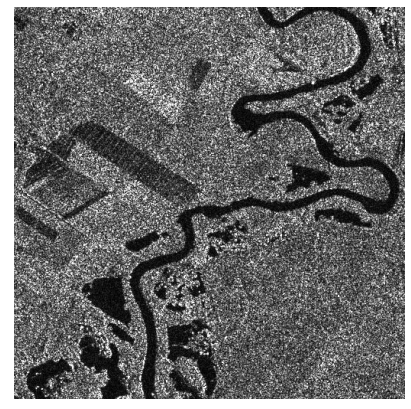

(a)

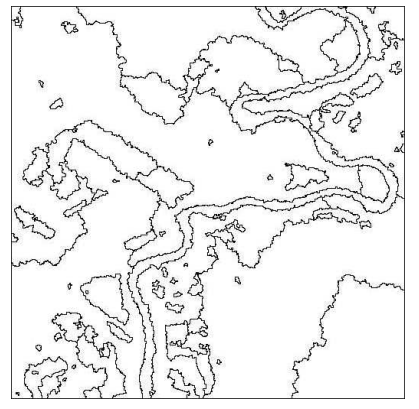

(b)

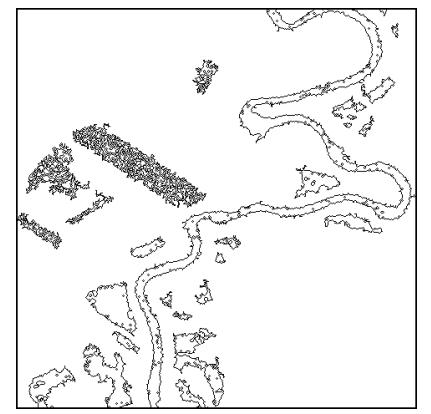

(c)

Figure 10. (a) Detail of the image Caçapava, 1 look, amplitude (b)edge map with 103 segments and (c) edge map of segmentation result using ENVI software.

contrast with neighboring regions.

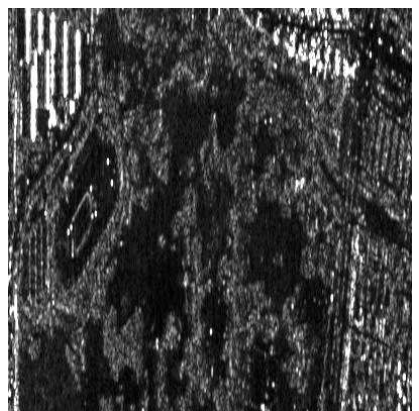

(a)

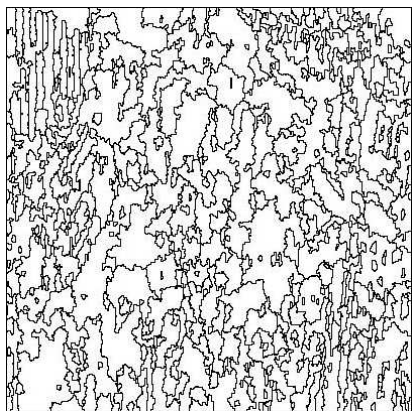

(b)

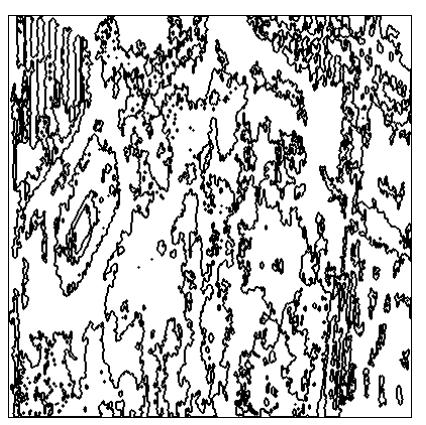

(c)

Figure 11. (a) Pedazo and (b) edge maps with 515 segments $\left(p_{0}=10^{-7}\right)$ and $(c)$ edge map of segmentation result using ENVI software.

Fig. 12 shows the edge map obtained from a $256 \times$ ) 256 subimage image Tapajós (3 looks and amplitude format) comprising 119 regions. This SAR image was taken on June 26, 1993 over Tapajós, Pará, Brazil, by JERS-1. The significance level $\left(p_{0}=10^{-5}\right)$ we adopted favored small fragments formation and the diagonal strip consists of two parts, which may be eliminated by diminishing the value of $p_{0}$.

\section{Concluding Remarks}

Research on developing SAR image segmentation algorithms is still a challenging issue to be explored. The difficulties in segmenting SAR images reside mainly on the presence of speckle noise. This paper provides a segmentation algorithm which is applied to SAR image, without speckle filtering preprocessing. Firstly, our approach divides an image into a great number of small regions by a statistical region growing procedure and accomplished a statistical analysis of speckle by using the coefficient of variation analysis. The region growing algorithm creates an initial partition which is forward processed by the hierarchical merging technique.

The statistical region growing step is necessary to generate and process conveniently the initial regions in order to provide input information for the merging 


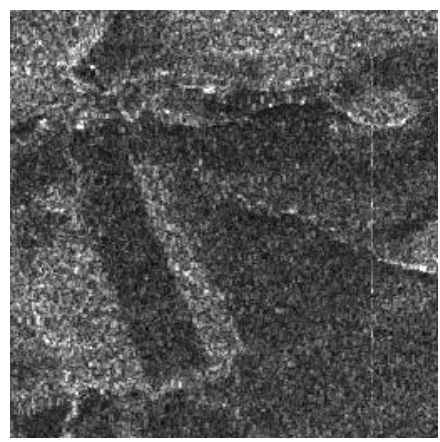

(a)

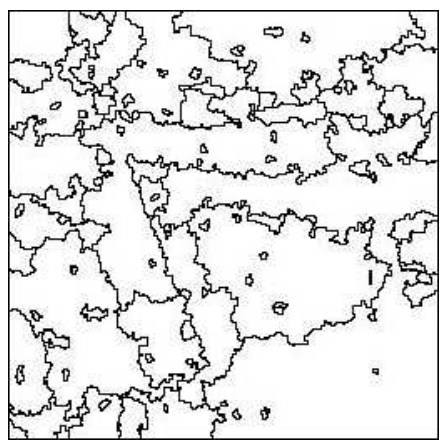

(b)

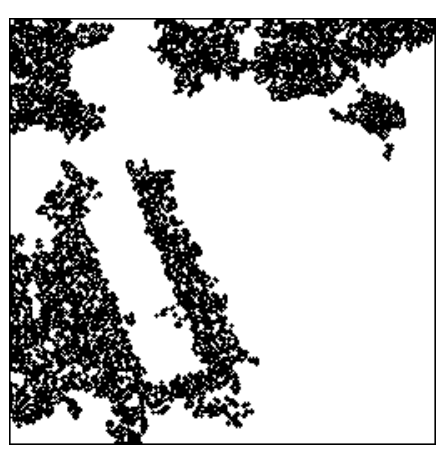

(c)

Figure 12. (a) SAR image Tapajos and (b) edge map with 119 segments and (c) edge map of segmentation result using ENVI software.

process, thus avoiding simple image tessellation. The region growing procedure based on the coefficient of variation favors the formation of the initial regions in homogeneous areas.

The proposed merging technique is a combination of hierarchical image analysis and hypothesis test. The former is implemented based on a cost function that uses the features of the regions to be merged, in addition to local ratio of means analysis. The two samples Kolmogorov-Smirnov (KS) hypothesis test is utilized to effective provide the merging a pair of regions, elected in the hierarchical region adjacency graph. Hierarchi- cal analysis favors a coordinated process, whereas regions are merged based on the statistical distribution of raw pixels .

The user can interact with the segmentation process by indicating the parameters: 1) the maximum of pixels (maxpixels) for the regions during the growing process (this can be left also as an internal parameter of the algorithm); 2) the number of looks $(n)$ in which the radar image was processed and 3) the significance level of the KS test $\left(p_{0}\right)$ set by the user, which controls the amount of regions present in the segmented image.

Further studies can include the following issues:

- The evaluation methodology can include other techniques that process filtered SAR images, as well as the comparison with current SAR segmentation approaches that work on non filtered SAR images, in order to improve the presented algorithms;

- This algorithm may be applied in environment monitoring which use SAR image such as coastal monitoring and oil spills detection in areas under petroleum exploitation.

- In situations which it is quite important to identify land use, e.g crops, forest or urban areas the region growing algorithm can be adjusted to the distributions displayed in .

The contribution of this paper concerns a new approach to perform image segmentation using the raw pixels as input data, without preprocessing. This methodology eliminates the filtering step, as well as it favors fine structures detection in speckled images.

\section{Acknowledgments}

This work was developed with partial financial support from CNPq, Brazil and from Applied Mathematical Science subprogram of the Office of Energy Research, U.S. Department of Energy, under Contract Number DE-AC03-76SF00098.* We wish to acknowledge Prof. Dr. Alejandro Frery for providing synthetic images. The authors also gratefully acknowledge the valuable contributions of the referees.

\section{References}

[1] D. T. Kuan, A. A. Sawchuck, T. C. Strand, P. Chavel, Adaptive noise smoothing filter for images with signal-dependent noise, IEEE Trans. Pattern Anal. and Mach. Intell. 07 (1985) 165177.

\footnotetext{
*Work was supported by the U.S. Department of Energy under Contract No. DE-AC02-05CH11231.
} 
[2] J. S. Lee, Digital image enhancement and noise filtering by use of local statistics, IEEE Trans. Pattern Anal. and Mach. Intell. 02 (1980) 165-168.

[3] F. N. S. Medeiros, N. D. A. Mascarenhas, L. F. Costa, Evaluation of speckle noise map filtering algorithms applied to SAR images, Int. J. of Remote Sens. 24 (24) (2003) 5197-5218.

[4] A. C. Frery, E. S. Almeida, G. T. Silva, F. N. S. Medeiros, E. L. P. Gouveia, K. M. Costa, Edge preserving noise reduction via combination of filters, in: XI RPIC - Reunión de Trabajo en Procesamiento de la Información y Control, 2005, pp. 620-625, Rio Cuarto, Argentina.

[5] R. C. P. Marques, E. A. Carvalho, R. C. S. Costa, F. N. S. Medeiros, Filtering effects on SAR images segmentation, in: Proc. of 11th Int. Conf. on Telecom. ICT'04, Vol. 3124 of Lecture Notes in Comput. Sci., Springer-Verlag, Germany, 2004, pp. 1041-1046.

[6] J. Lemoigne, J. C. Tilton, Refining image segmentation by integration of edge and region data, IEEE Trans. Geosci. and Remote Sens. 33 (3) (1995) 605-615,.

[7] R. Touzi, A. Lopès, P. Bousquet, A statistical and geometrical edge detector for SAR images, IEEE Trans. Geosci. and Remote Sens. 26 (06) (1988) 764-773.

[8] A. Lopès, R. Fjørtoft, D. Ducrot, P. Marthon, C. Lemarèchal, Edge detection and segmentation of SAR images in homogeneous regions, in: Information Process. for Remote Sens., World Scientific Publishing Co., Singapore, 1999, p. 139.

[9] Z. Bai, P. He, An improved ratio edge detector for target detection in SAR images, in: Proc. of IEEE Int. Conf. on Neural Networks \& Signal Process., Nanjing, China, 2003, pp. 982-985.

[10] M. Airouche, M. Zelmat, M. Kidouche, Statistical edge detectors applied to sar images, in: Int. J. of Computers, Communications and Control, Vol. 3 of Suppl. issue: Proceedings of ICCCC 2008, 2008, pp. 144-149.

[11] Q. Yu, D. A. Clausi, Sar sea-ice image analysis based on iterative region growing using semantics, IEEE Trans. Geosci. and Remote Sens. 45 (12) (2007) 3919-3931.

[12] G. P. Bernad, L. Denise, P. Réfrégier, Hierarchical feature-based classification approach for fast and user-interactive SAR image interpretation, IEEE Geosci. and Remote Sens. Letters 6 (1) (2009) 117-121.

[13] F. Galland, N. Bertaux, P. Réfrégier, Minimum description length synthetic aperture radar image segmentation, IEEE Trans. Image Process. 12 (09) (2003) 995-1006.

[14] R. Cook, I. McConnel, C. Oliver, E. Welbourne, MUM (Merge Using Moments) segmentation for SAR images, in: Proc. of Europto Conf. on SAR Data Process. for Remote Sens., SPIE, Vol. 2316, 1994, pp. 92-103, Rome, Italy.

[15] Y. Yang, H. Sun, C. He, Supervised SAR image MPM segmentation based on region-based hierarchical model, IEEE Geosci. and Remote Sens. Letters 03 (04) (2006) 517-521.

[16] J. M. Beaulieu, J. M. Goldberg, Hierarchy in picture segmentation: A stepwise optimization approach, IEEE Trans. Pattern Anal. and Mach. Intell. 11 (02) (1989) 150-163.

[17] A. Plaza, J. Tilton, Automated selection of results in hierarchical segmentations of remotely sensed hyperspectral images, in: IEEE International Geoscience and Remote Sensing Symposium, Vol. 7 of Suppl. issue: Proceedings of ICCCC 2008, 2005, pp. 4946 - 4949.

[18] A. C. Frery, H. J. Müller, C. C. F. Yanasse, S. J. S. Sant'Anna, A model for extremely heterogeneous clutter, IEEE Trans. Geosci. and Remote Sens. 35 (03) (1997) 648-659.

[19] E. V. D. Lucca, C. C. Freitas, A. C. Frery, S. J. S. Sant'anna, Comparison of SAR segmentation algorithms, in: Proc. of Second Latino-American Seminar on Radar Remote Sens., Vol. 434, SantosSP, Brazil, 1998, pp. 123-130.

[20] A. Lopes, R. Touzi, E. Nezry, Adaptive speckle filters and scene heterogeneity, IEEE Geosci. and Remote Sens. Lett. 28 (6) (1990) 992-1000.

[21] R. Touzi, A review of speckle filtering in the context of estimation theory, IEEE Trans. Geosci. and Remote Sens. 40 (11) (2002) 2392-2404.

[22] J. M. Park, W. J. Song, W. A. Pearlman, Speckle filtering of SAR images based on adaptive windowing, IEE Proc. Vision, Image and Signal Process. 146 (04) (1999) 191-197. 
[23] A. Lopes, E. Nezry, R. Touzi, H. Laur, Structure detection and statistical adaptive speckle filtering in sar images, Int. J. of Remote Sens. 14 (9) (1993) 1735-1758.

[24] L. A. Frulla, J. A. Milovich, D. A. Gagliardini, Automatic computation of speckle standard deviation in SAR images, Int. J. of Remote Sens. 21 (15) (2000) 2883-2899.

[25] J. M. Beaulieu, Utilisation of segment border information in hierarchical segmentation, Technical Report, University of Laval, Dept. of Informatics, Quebec, Canada, [On line] available at http://www.casi.ca/papers/3-11.pdf, access date 12/10/04 (2004).

[26] G. B. Bénié, K. P. B. Thomson, Hierarchical image segmentation using local and adaptive similariry rules, Int. J. of Remote Sens. 13 (08) (1992) 15591570 .

[27] J. C. Tilton, Hierarchical image segmentation, Online Journal of Space Communication 3 (2003) 16.

[28] Q. Yu, D. A. Clausi, Irgs: Image segmentation using edge penalties and region growing, IEEE Trans. Patt. Analys. Mach. Intell. 30 (12) (2008) 2126-2139.

[29] R. Fjørtoft, A. Lopès, P. Marthon, E. CuberoCastan, An optimal multiedge detector for SAR image segmentation, IEEE Trans. Geosci. and Remote Sens. 36 (03) (1998) 793-802.

[30] B. J. Mealy, Fast multiphase region count reduction for partitioned images, Technical Report UCSC-CRL-02-40, University of California - Dept. of Comput. Engineering, Santa Cruz, USA, [On line] available at www.soe.ucsc.edu/research/reports/ucsc-crl-02-40.ps.Z, access date 05/11/05 (December 2002).

[31] W. Press, S. A. Teukolsky, W. T. Vetterling, B. P. Flannery, Numerical recipes in C: The art of scientific computing, 2nd Edition, Cambridge University Press, New York, 1992.

[32] M. Fisz, Probability theory and mathematical statistics, 3rd Edition, John Wiley \& Sons, New York, 1963.

[33] L. M. Delves, R. Wilkinson, C. J. Oliver, R. G. White, Comparing the performance of SAR image segmentation algorithms, Int. J. of Remote Sens. 13 (11) (1992) 2121-2149.
[34] Y. J. Zhang, A survey on evaluation methods for image segmentation, Pattern Recognition 29 (08) (1996) 1335-1346. 